\title{
ON CR-SUBMANIFOLDS OF THE SIX-DIMENSIONAL SPHERE
}

\author{
M. A. BASHIR \\ Mathematics Department, College of Science \\ King Saud University \\ P.O. Box 2455, Riyadh 11451 \\ Saudi Arabia
}

(Received July 13, 1993)

\begin{abstract}
We consider proper CR-submanifolds of the six-dimensional sphere $S^{6}$. We prove that $S^{6}$ does not admit compact proper CR-submanifolds with non-negative sectional curvature and integrable holomorphic distribution.
\end{abstract}

KEY WORDS AND PHRASES. CR-submanifolds, Kaehler manifold, nearly Kaehler manifold, the six-dimensional sphere, almost complex structures.

1991 AMS SUBJECT CLASSIFICATION CODES. Primary, 53C40; Secondary 53C55.

1. INTRODUCTION. The study of CR-submanifolds of a Kaehler manifold was initiated by Bejancu [1]. This study generalizes both the complex submanifolds as well as the totally real submanifolds. For this reason, it has become the subject of interest to many mathematicians [3]. Of all the Euclidian spheres, only $S^{2}$ and $S^{6}$ admit the almost complex structure of which $S^{2}$ is complex and $S^{6}$ is not. It is known that $S^{6}$ is an almost hermitian manifold which is nearly Kaehler but not Kaehler, that is, the almost complex structure is not parallel with respect to the Riemannian connection on $S^{6}$ [4]. CR-submanifolds of $S^{6}$ have been studied by several mathematicians. For instance Sekigawa [7] proved that $S^{6}$ does not contain any CR-product submanifold. Gray [5] has shown that $S^{6}$ does not admit a 4-dimensional complex submanifold.

In this paper, we consider compact proper CR-submanifolds of $S^{6}$. We obtain the following:

THEOREM. $S^{6}$ does not admit any compact proper CR-submanifold with non-negative sectional curvature and integrable holomorphic distribution.

2. PRELIMINARIES. Let $C$ be the set of all purely imaginary Cayley numbers. $C$ can be viewed as a 7-dimensional linear subspace $\mathbb{R}^{7}$ of $\mathbb{R}^{8}$. Consider the unit hypersurface which is centered at the origin:

$$
S^{6}(1)=\{x \in C:\langle x, x\rangle=1\}
$$

The tangent space $T_{x} S^{6}$ of $S^{6}$ at a point $x$ may be identified with the affine subspace of $C$ which is orthogonal to $x$. $A(1,1)$ tensor field $J$ on $S^{6}$ is defined by

$$
J_{x} U=X \times U
$$

where the above product is defined as in [4] for $x \varepsilon S^{6}$ and $U \varepsilon T_{x} S^{6}$. The tensor field $J$ determines an almost complex structure (i.e., $J^{2}=-i d$ ) on $S^{6}$. If $\bar{\nabla}$ is the Riemannian connection on $S^{6}$, then $\left(\bar{\nabla}_{X} J\right) X=0$ for any $X \varepsilon \mathfrak{E}\left(S^{6}\right)$, i.e., $S^{6}$ is nearly Kaehler. 
$A(2 p+q)$-dimensional submanifold $M$ of $S^{6}$ is called a CR-submanifold if there exists a pair of orthogonal complementary distribution $D$ and $\frac{1}{D}$ such that $J D=D$ and $J \frac{1}{D} \varepsilon \nu$ where $\nu$ is the normal bundle of $M$. The distributions $D$ and $\frac{1}{D}$ are called the holomorphic distribution and the totally real distribution respectively with $\operatorname{dim} D=2 p$ and $\operatorname{dim} D^{\perp}=q$. The normal bundle $\nu$ splits as $\nu=J \frac{1}{D} \oplus \mu$ where $\mu$ is invariant sub-bundle of $\nu$ under $J$. The CR-submanifold is said to be proper if neither $D=\{0\}$ nor $\frac{1}{D}=\{0\}$. A proper CR-submanifold $M$ of $S^{6}$ is said to be a CR-product submanifold if it is locally the Riemannian product of a holomorphic submanifold and a totally real submanifold of $S^{6}$. It is known that there does not exist any CR-product submanifolds in $S^{6}[7]$.

Let $\nabla$ be the Riemannian connection on $(M, g)$ where $g$ is the induced metric. Then the curvature tensor $R$ of $(M, g)$ of type $(1,3)$ is defined by

$$
R(X, Y) Z=\nabla_{X} \nabla_{Y} Z-\nabla_{Y} \nabla_{X} Z-\nabla_{[X, Y]} Z, \quad X, Y, Z \varepsilon \mathfrak{X}(M)
$$

The sectional curvature $K(X, Y)$ of the plane section determined by $\{X, Y\}$ is defined by

$$
K(X, Y)=R(X, Y, Y, X)\left\{\|X\|^{2}\|Y\|^{2}-g(X, Y)^{2}\right\}^{-1} \text { where } R(X, Y, Z, W)=g(R(X, Y) Z, W) .
$$

The Ricci tensor of $(M, g)$ is defined by

$$
\operatorname{Ric}(X, Y)=\sum_{i=1}^{n} R\left(e_{i}, X, Y, e_{\imath}\right), \quad X, Y \varepsilon \mathfrak{X}(M)
$$

where $\left\{e_{1}, \cdots, e_{n}\right\}$ is a local orthonormal frame field on $M$. On a compact Riemannian manifold the following integral formula holds for any $X \varepsilon \mathfrak{X}(M)$ (cf [8]).

$$
\int_{M}\left\{\operatorname{Ric}(X, X)+\|\nabla X\|^{2}-\frac{1}{2}\|d \eta\|^{2}-(\operatorname{div} X)^{2}\right\} d v=0
$$

where $\eta$ is a 1 -form dual to $X$, i.e., $g(X, Z)=\eta(Z)$, for

$$
Z \varepsilon \mathfrak{X}(M) \text { and }\|\nabla X\|^{2}=\sum_{i=1}^{n} g\left(\nabla_{e_{i}} X, \nabla_{e_{i}} X\right) .
$$

Let $h$ be the second fundamental form. $M$ is said to be totally geodesic if $h \equiv 0$ and $M$ is said to be totally umbilical if $h(X, Y)=g(X, Y) H$ where $H$ is the mean curvature tensor defined by $H=\frac{1}{n}$ trace $h$.

\section{PROOF OF THE THEOREM.}

Since $D$ is integrable, then the integral submanifold of the distribution $D$ is a Kaehler manifold. Since $M$ is proper then $\operatorname{dim} D=4$ is ruled out by a result of Gray [5] namely $S^{6}$ does not contain a 4-dimensional complex submanifold. Therefore $\operatorname{dim} D=2$. Since $\nu=J \frac{1}{D} \oplus \mu$ and $M$ is a proper CR-submanifold of $S^{6}$ we have $\operatorname{dim} \frac{1}{D}=1$, i.e., $M$ is 3 -dimensional. Now let $w$ be a 2 -form on the integral submanifold of $D$ and let $\eta$ be its dual. Since the integral submanifold of $D$ is Kaehler, $w$ is harmonic (cf. [6]). Using Poincare duality theorem, its dual $\eta$ is also harmonic, i.e., $d \eta=\delta \eta=0$.

Now from the hypothesis of the theorem, we get $\operatorname{Ric}(Z, Z) \geq 0$. Using the integral formula on this page and $Z \varepsilon \stackrel{1}{D}$ we have

$$
\int_{M}\left\{\operatorname{Ric}(Z, Z)-\frac{1}{2}\|d \eta\|^{2}+\|\nabla Z\|^{2}-(\delta \eta)^{2}\right\} d v=0
$$


from which we get $\nabla_{X} Z=0$ for all $X \in \mathfrak{X}(M)$ and $Z \varepsilon \dot{D}$, i.e., the distribution $\frac{1}{D}$ is parallel. Also $g(Y, Z)=0$ for all $Y \varepsilon D$ gives $\nabla_{X} Y=0$ for all $X \varepsilon \mathfrak{X}(M)$ and $Y \varepsilon D$. This means that $D$ is also parallel. $D$ and $\frac{1}{D}$ being parallel implies that $M$ is a CR-product, which is a contradiction to the fact that $S^{6}$ does not have any CR-product submanifold [7]. Therefore our theorem is proven.

COROLLARY 1. There does not exist a compact totally umbilical proper CR-submanifolds of $S^{6}$ with integrable distribution $D$.

PROOF. Since $S^{6}$ is of constant positive curvature, the curvature tensor $\bar{R}$ of $S^{6}$ is given by $\bar{R}(X, Y, Z, W)=c\{g(X, W) g(Y, Z)-g(Z, X) g(Y, W)\}$. Using this in Gauss equation

$$
R(X, Y, Z, W)=\bar{R}(X, Y, Z, W)+g(h(X, W), h(Y, Z))-g(h(Z, X), h(Y, W))
$$

with the assumption that $M$ is totally umbilical (i.e., $h(X, Y)=g(X, Y) H$ ) we get $R(X, Y, Y, X)=c+\|H\|^{2}>0 \cdot X, Y \varepsilon \mathfrak{X}(M)$. This implies that $M$ is of positive sectional curvature. Then the corollary follows from the theorem.

COROLLARY 2. There does not exist a compact totally geodesic proper CR-submanifold of $S^{6}$ with integrable distribution $D$.

PROOF. Since $M$ is totally geodesic in $S^{6}$, then it follows immediately from Gauss equation that $M$ is of positive sectional curvature. Thus the corollary follows from the theorem.

REMARK. If $\operatorname{dim} M=3$, then Corollary 1 holds without the assumption that $D$ is integrable. This is a result proved previously by Bashir [2].

\section{REFERENCES}

1. BEJANCU, A., CR-submanifolds of a Kaehler manifold, Proc. Amer. Math. Soc. 69 (1978), 135-142.

2. BASHIR, M., On the three-dimensional CR-submanifolds of the six-dimensional sphere, Internat. J. Math. and Math. Sci. 14 (1991), 675-678.

3. CHEN, B.Y., CR-submanifolds of a Kaehler manifold I and II, J. Diff. 16 (1981), 305-322 and 493-502.

4. FUKAMI, T. and ISHIHARA, S., Almost Hermitian structure on $S^{6}$, Tohoku Math. J. 7 (1955), 151-156.

5. GRAY, A., Almost complex submanifolds of six sphere, Proc. Amer. Math. Soc. 20 (1969), 277-279.

6. GOLDBERG, S., Curvature and Homology, Academic Press, New York, 1962.

7. SEKIGAWA, K., Some CR-submanifolds in a 6-dimensional sphere, Tensor, N.S. 41 (1984), 13-20.

8. YANO, K. and BOCHNER, S., Curvature and Betti numbers, Ann. of Math. Stud. 32, 1953. 


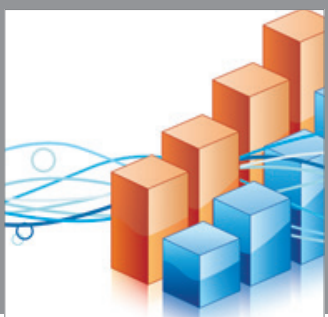

Advances in

Operations Research

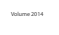

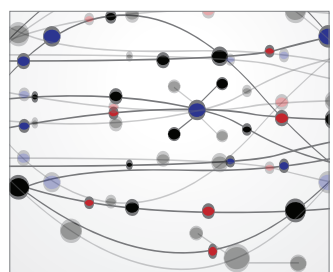

\section{The Scientific} World Journal
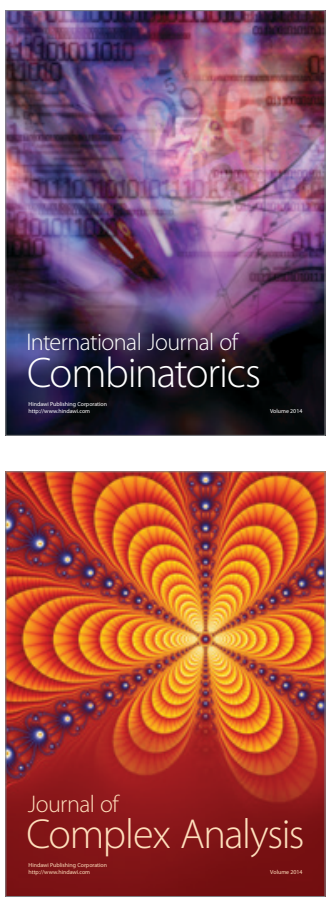

International Journal of

Mathematics and

Mathematical

Sciences
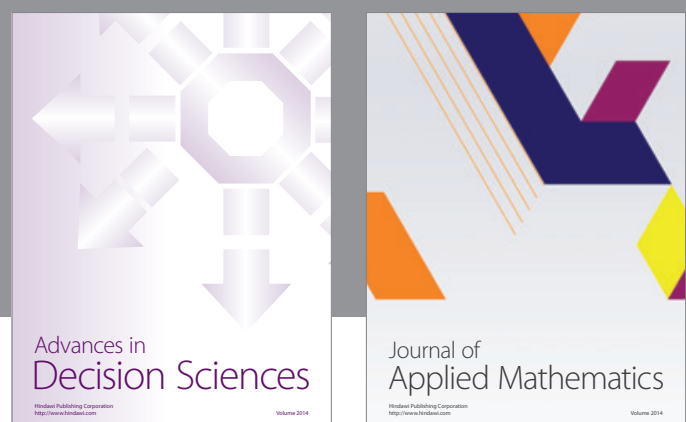

Journal of

Applied Mathematics
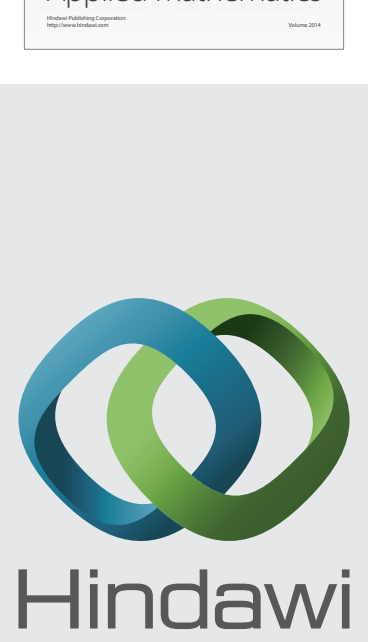

Submit your manuscripts at http://www.hindawi.com
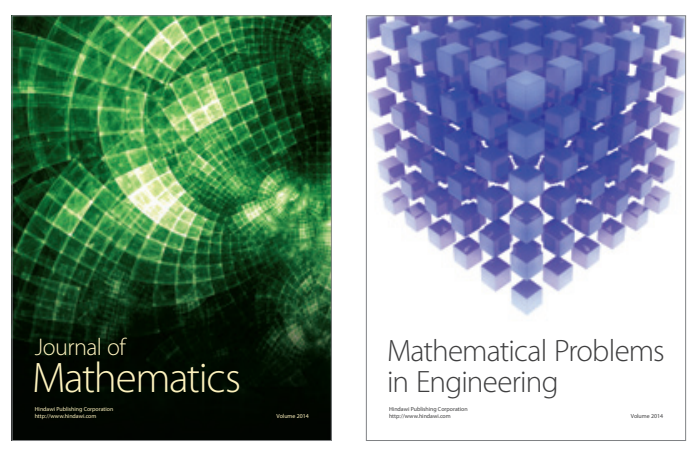

Mathematical Problems in Engineering
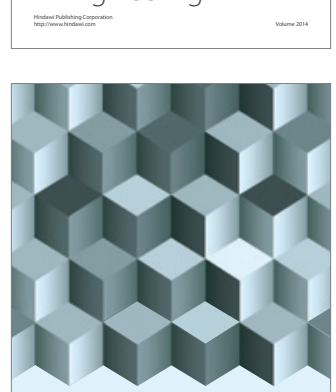

Journal of

Function Spaces
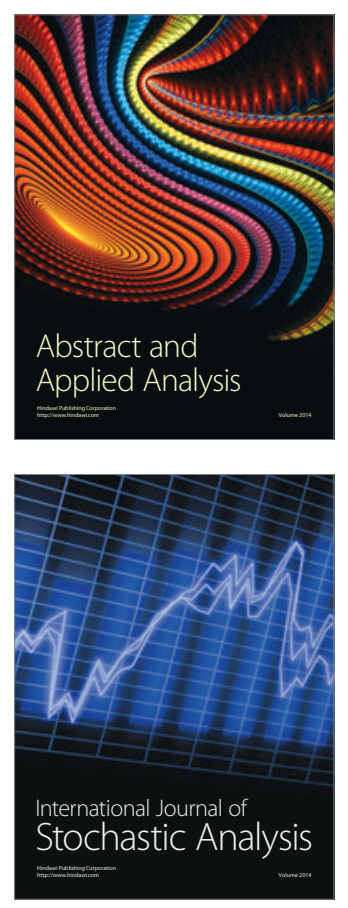

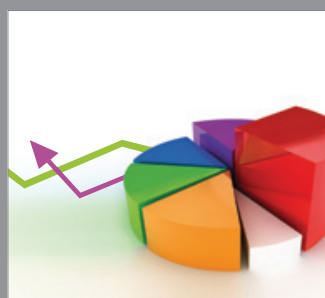

ournal of

Probability and Statistics

Promensencen
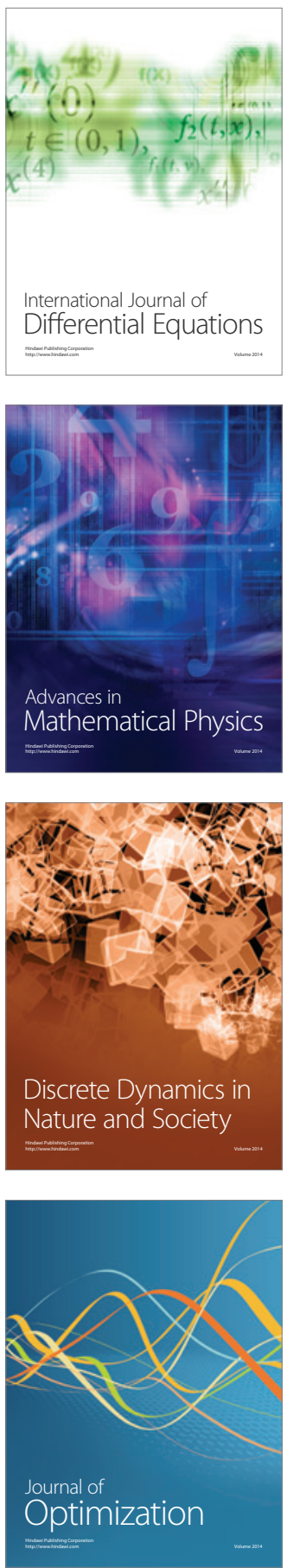